\title{
El análisis visual de los estudios de integración paisajística a partir de datos LiDAR
}

\author{
Collado Latorre, J.C. ${ }^{* 1}$, Colomer Sendra, V.C. ${ }^{2}$, Martínez Cortijo, F.J. ${ }^{3}$ \\ ${ }^{1}$ Calle Valencia $n .{ }^{\circ} 6$ Camporrobles C.P. 46330 \\ 2 Departamento de Urbanismo. Universitat Politècnica de València. Calle Camino de Vera (s/n) C.P. 46022 \\ ${ }^{3}$ Departamento Ingeniería Rural y Agroalimentaria. Universitat Politècnica de València. Calle Camino de Vera ( $\mathrm{s} / \mathrm{n}$ ) \\ “Autor para correspondencia: jucolla@doctor.upv.es
}

\section{Resumen}

Con el presente trabajo se pretende constatar la viabilidad y mayor exactitud que supone el empleo de la tecnología de datos LiDAR a la hora de obtener un modelo digital del terreno más realista, con su empleo en un software en el campo de los Sistemas de Información Geográfica, que permita la edición, análisis, tratamiento, diseño e impresión a partir de estos datos. En el trabajo se constata la diferencia y clara mejora que supone el empleo de esta nueva tecnología con respecto a los datos que se utilizaban para estas aplicaciones hasta ahora. Así se obtienen resultados mucho más realistas y precisos que permiten realizar trabajos y obtener conclusiones que se adaptan mucho más las necesidades específicas como es el caso de la obtención de cuencas visuales en estudios de integración paisajística.

Palabras clave: SIG, LiDAR, DSM (Modelo Digital de Superficie), Integración Paisajística. 


\section{Introducción}

El objetivo de esta investigación es el uso de modelos digitales de superficie en la elaboración del análisis visual de los estudios de integración paisajística a partir de datos LiDAR (Light detection and ranging). Los datos LiDAR han sido extraídos de la página web del Centro de Descargas del Centro Nacional de Información Geográfica (CNIG), estos datos fueron capturados en el año 2009 con un sensor Leica ALS60 de pulsos discretos y espejo oscilante. Los datos son un producto gratuito, con una densidad de 0,5 puntos por metro cuadrado (con una separación media entre puntos de $1.4 \mathrm{~cm}$ ). La precisión altimétrica es de $20 \mathrm{~cm}$. Se encuentran en ficheros cuadrados de tamaño $2 \times 2 \mathrm{~km}$ en formato LAZ (archivo LAS comprimido), el sistema geodésico de referencia es el ETRS89. Estos datos proporcionan modelos digitales de superficie de mayor exactitud y precisión con la consecuente obtención de cuenca visuales también con estas características ya que se elaboran a partir de estos modelos con sistemas de información geográfica. Como caso práctico se ha realizado el análisis visual de la realización del parque eólico Sierra Negrete II de la Zona 9 del Plan Eólico de la Comunitat Valenciana ubicado en el Monte de utilidad pública N. ${ }^{\circ} 95$ (Fig. 1).

En la actualidad se han calculado cuencas visuales a partir de modelos digitales de superficie con sistemas de información geográfica (SIG) para mejorar el modelo de tráfico en una vía verde urbana (Lindsey et al., 2008), para realizar modelos visuales con las características de rutas peatonales urbanas (Wilson et al.,2008), para para medir el valor de casas a partir de un modelo de precios de la propiedad (Hamilton y Morgan, 2010), para la representación de corto alcance de la visibilidad obtenida afectada por las obstrucciones troncales de los árboles (Murgoitio et al., 2013), para evaluar la idoneidad de los modelos digitales de superficie variando los niveles de detalle(Klouček et al., 2015), para el estudio de estudiaron la colocación de señales de evacuación que indican a los lugareños y visitantes la ruta que deben tomar en caso de riesgo de tsunamis (Lonergan et al., 2015).

Los estudios de integración paisajística son instrumentos del paisaje en la normativa de paisaje de las comunidades autónomas de España. Según la normativa de las comunidades autónomas estos instrumentos del paisaje de integración paisajística tienen distinta denominación, aunque el objetivo es el mismo, a continuación se recoge la definición que está incluida en la normativa de paisaje de cada comunidad:

Cataluña (Decreto 343 del 2006, por el que se desarrolla la Ley 8 del 8 de junio del 2005, de Protección, Gestión y Ordenación del Paisaje, y se regulan los estudios e informes de impacto e integración paisajística): El estudio de impacto e integración paisajística es un documento técnico destinado a considerar las consecuencias que tiene sobre el paisaje la ejecución de actuaciones, proyectos de obras o actividades y a exponer los criterios adoptados para su integración.

Cantabria (Ley 4/2014, de 22 de diciembre, del Paisaje. Cantabria: Diario Oficial de Cantabria): Los Análisis de Impacto e Integración Paisajística son documen- 


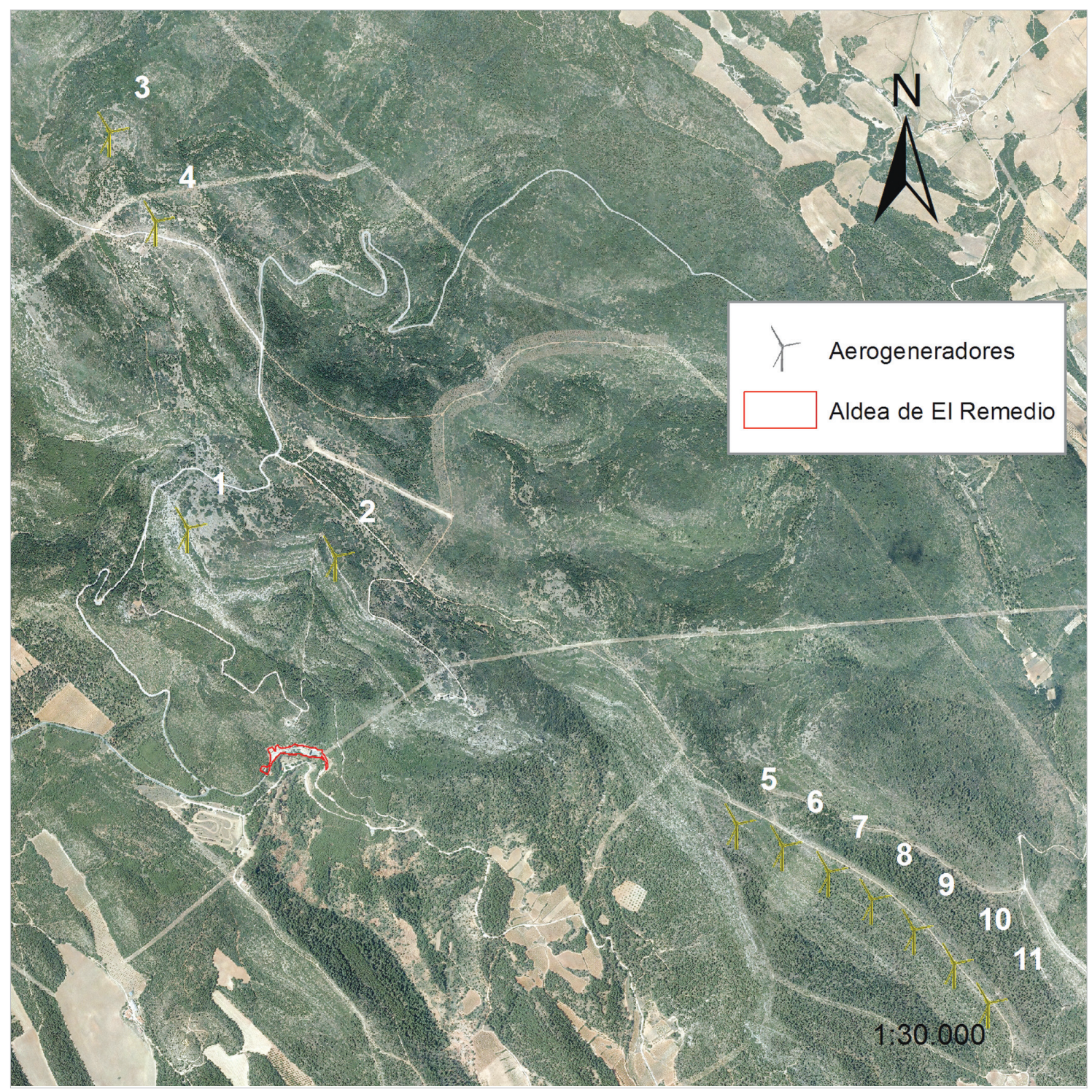

Figura 1. Ubicación de los aerogeneradores y de la aldea.

tos técnicos que tienen por objeto valorar y cuantificar la magnitud y la importancia de los efectos que una actuación puede llegar a producir en el paisaje y en su percepción, y proponer las medidas adecuadas para evitar los impactos o mitigar los posibles efectos negativos.

Comunitat Valenciana (Ley 5/2014, de 25 de julio, de la Generalitat, de Ordenación del Territorio, Urbanismo y Paisaje, de la Comunitat Valenciana): Los estudios de integración paisajística, que valoran los efectos sobre el carácter y la percepción del paisaje de planes no sometidos a evaluación ambiental y territorial estratégica, así como de proyectos y actuaciones con incidencia en el paisaje y establecen medidas para evitar o mitigar los posibles efectos negativos. 
Galicia (Ley 7/2008, de 7 de julio, de protección del paisaje de Galicia): En todos los proyectos que deban someterse al procedimiento de Declaración de impacto ambiental, según se establece en la legislación sectorial vigente, las entidades promotoras habrán de incorporar en el estudio de impacto ambiental un estudio de impacto e integración paisajística, documento específico en el que se evaluarán los efectos e impactos que el proyecto pueda provocar en el paisaje y las medidas de integración paisajística propuestas por dichas entidades.

Murcia (Ley 13/2015, de 30 de marzo, de ordenación territorial y urbanística de la Región de Murcia): El Estudio de Impacto Territorial es un documento técnico complementario de los instrumentos de ordenación territorial y de los de planeamiento para los que así se prevea en esta ley. El Estudio de Impacto Territorial comprenderá los estudios y análisis para predecir, valorar y corregir el posible impacto sobre la estructura territorial y los impactos sectoriales.

País Vasco (Decreto 90/2014, de 3 de junio, sobre protección, gestión y ordenación del paisaje en la ordenación del territorio de la Comunidad Autónoma del País Vasco. País Vasco: Diario Oficial del País Vasco): Los Estudios de integración paisajística son los documentos técnicos destinados a considerar las consecuencias que tiene sobre el paisaje la ejecución de proyectos de obras y actividades, así como a exponer los criterios y las medidas adoptadas para la adecuada integración de las obras y actividades en el paisaje.

Todos estos estudios se basan en la realización de un análisis de la visibilidad, del que no se incluye estas normativas de paisaje una metodología para su realización, siendo a elección del paisajista el método a seguir para la elaboración de este.

\section{Material y métodos}

\subsection{Materiales}

Los materiales utilizados para el desarrollo de esta investigación son los siguientes:

Ordenador con las características Intel ${ }^{\circledR}$ Core $^{\mathrm{TM}}$ i7-6500U con Intel ${ }^{\circledR}$ HD Graphics 520 (2,5 GHz, hasta 3,1 GHz, $4 \mathrm{MB}$ de caché, 2 núcleos),

Software ArcGIS 10.1.

Archivos en formato LAZ de la zona de estudio.

Archivo ESRI shapefile de la delimitación de la aldea de El Remedio.

Archivo ESRI shapefile de la ubicación del parque eólico Sierra Negrete II.

\subsection{Metodología}

2.2.1 Obtención del modelo digital de superficie 
En primer lugar, se descomprimieron los archivos en formato LAZ a formato LAS, se utilizó el programa gratuito LasTask proporcionado por el Centro de Descargas del CNIG que los descomprime todos los archivos a la vez.

Para obtener la nube de puntos del área del municipio analizado se utilizó la aplicación LiDARToolKit del programa FUSION v. 3.0.3 (McGaughey, 2009), con este mismo programa los archivos en formato LAS se unieron y se recortaron respecto al área de estudio.

Posteriormente se aplicó un filtro al archivo LAS del área de estudio para eliminar la existencia de ruido (outlayers). El ruido son aquellos puntos determinados erróneamente (aves, objetos, etc.). La cuenca visual realizada con un modelo digital de superficie con outlayers tendrá errores ya que estos aparecen como elementos lineales que interfieren en las líneas de visión del observador. En la figura 2 siguiente puede verse la influencia de los outlayers al generar el modelo digital de superficie.

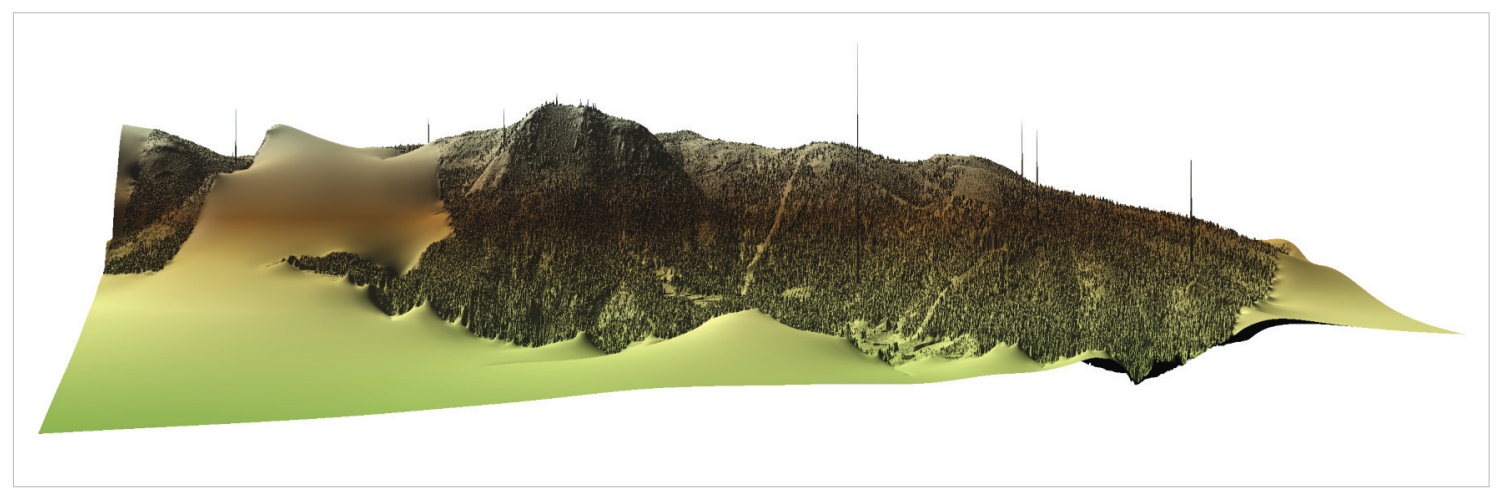

Figura 2. Representación de outlayers en el modelo digital de superficie.

Tras realizar los pasos anteriores se procedió a la obtención de los primeros retornos de la nube de puntos separándolos de los últimos retornos con la herramienta FirstLastReturn para realizar los modelos digitales de superficie (DSM). Los DSM se originan a partir de los primeros pulsos del láser, los cuales representan la superficie topográfica. Los puntos del primer pulso se reflejan desde la superficie superior, que puede ser de la vegetación, de los edificios o del terreno. El modelo digital de superficie (DSM) se obtiene tomando el valor más alto de todos los puntos dentro de cada píxel y luego se interpola para obtener los valores de los píxeles a los que les faltan estos valores.

Para utilizar la nube de puntos obtenida del programa FUSION en el programa ArcGISse creó un nuevo archivo en formato ESRI Shapefile de puntos a partir del archivo en formato LAS, debido a que este programa no permite trabajar con los datos en formato LAS a la hora de usar las herramientas de los métodos de interpolación de creación de modelos digitales.

Los puntos en formato ESRI Shapefile obtenidos en la capa anterior se encuentran en "entidad multiparte", así se denomina a las capas que tienen más de una parte 
física pero que sólo hacen referencia a un conjunto de atributos en la base de datos, por lo que en cada registro de la tabla de atributos de la entidad están incluidos varios puntos. Para poder utilizar el valor de capa punto individualmente se han separado los puntos obteniendo los atributos de cada punto (elevación).

A partir de la capa con los puntos LiDAR se realizó el modelo digital de superficie con el método de interpolación "Topo to raster" incluido en la barra de herramientas "SpatialAnalyst”. Debido a que se quiere obtener la máxima precisión en el modelo digital de superficie se utilizaron todos los puntos LiDAR y la resolución de celda de la malla elegida ha sido de $1 \mathrm{~m}$. Al realizar el modelo digital de superficie puede verse una zona lisa debido la falta de puntos LiDAR debido a un error en la toma o tratamiento de los datos, en este caso el programa ha interpolado las elevaciones con las colindantes.

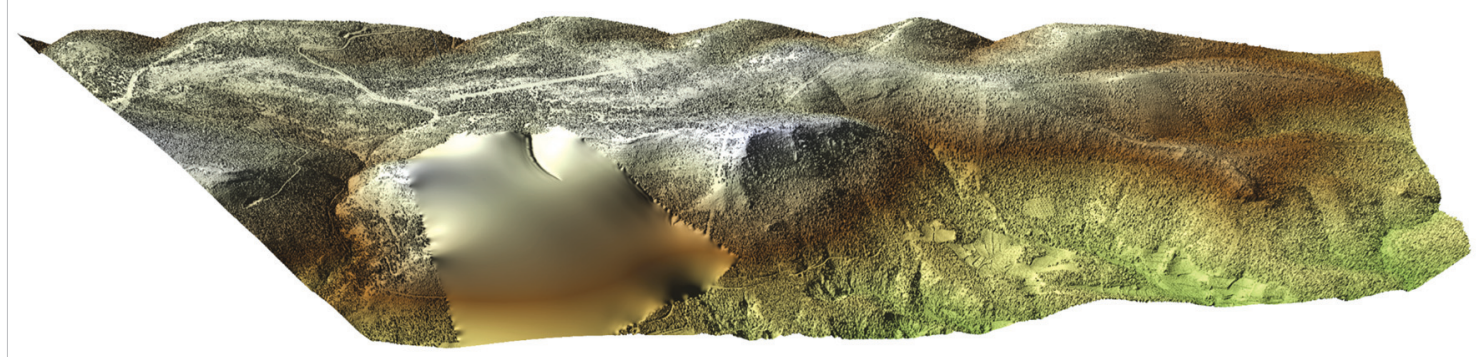

Figura 3. Representación del modelo digital de superficie de la zona de estudio.

\subsection{Análisis visual con ArGIS 10.1}

Para realizar el análisis de visibilidad se ha utilizado la herramienta "ObserverPoints" del programa ArcGIS 10.1 para obtener la cuenca visual. En este caso la cuenca visual realizada es del tipo inversa descrita por Fisher (1996), tiene los mismos principios que el análisis de cuenca visual, sin embargo se utiliza para determinar la visibilidad de un punto objetivo determinado a varios puntos de observación. En este caso los puntos objetivo son los aerogeneradores con una altura de 80 metros y el punto de observación la aldea de El Remedio (Utiel) con una altura de 1,65 m, correspondiente a la altura de una persona.

\section{Resultados}

En comparación con un modelo digital del terreno con tamaño de celda $10 \mathrm{~m}$ realizado a partir de curvas de nivel cada 5 metros se observa que el nivel de detalle del paisaje es mayor en el modelo digital de superficie, con lo que la interpretación del paisaje es mayor (Figs. 4 y 5). 


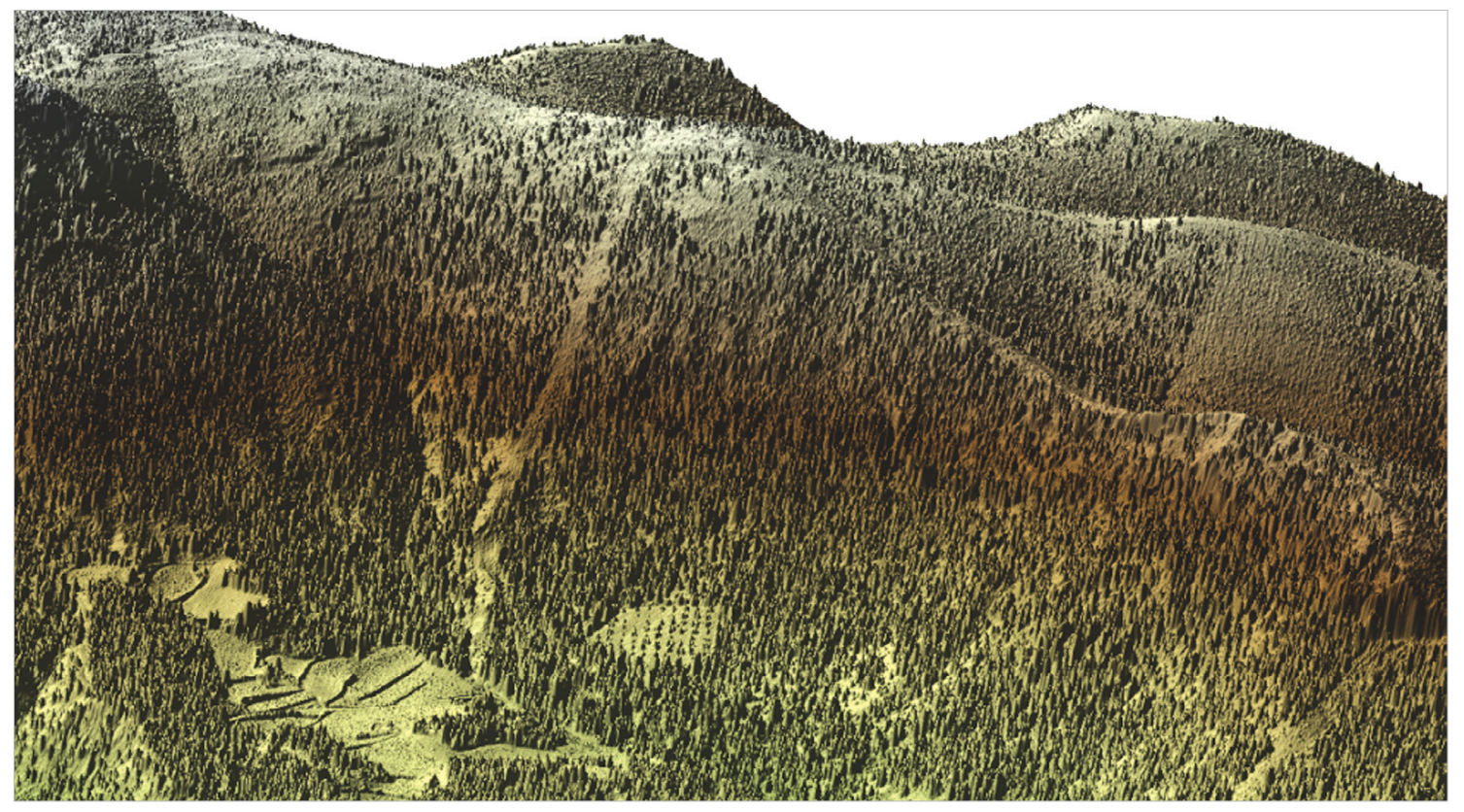

Figura 4. Representación del MDS con mayor detalle.

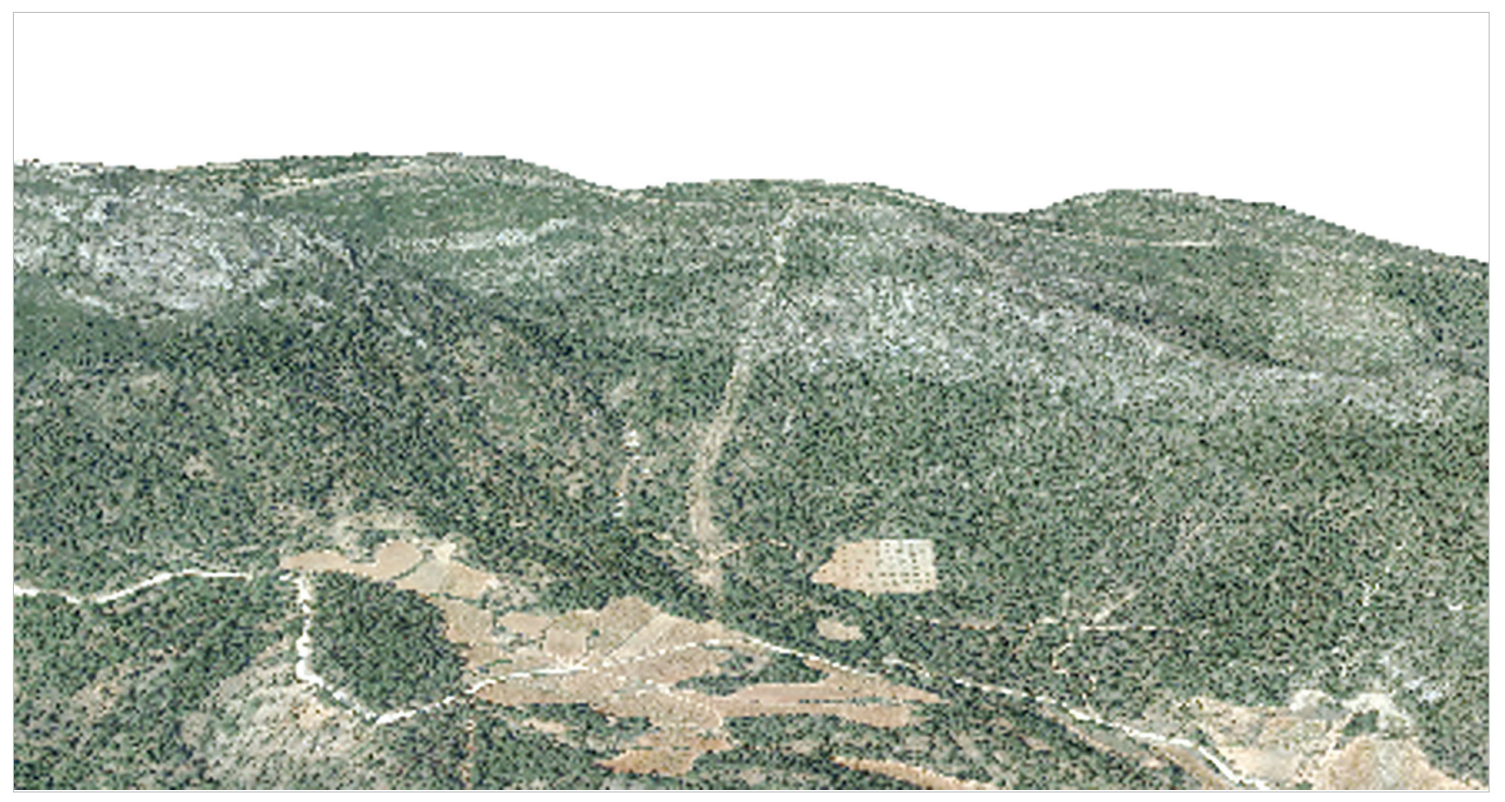

Figura 4. Representación del MDS con mayor detalle.

Para determinar la visibilidad en la aldea de El Remedio de los aerogeneradores se ha recortado el área de la cuenca visual obtenida respecto de los límites de la aldea, donde se obtiene una tabla en el archivo ráster con varias zonas según el número de aerogeneradores visibles en el área delimitada por ésta.

Como resultado final del análisis visual se ha obtenido que únicamente los aerogeneradores 1 y 2 son visibles desde la aldea de El Remedio (Fig. 6). 


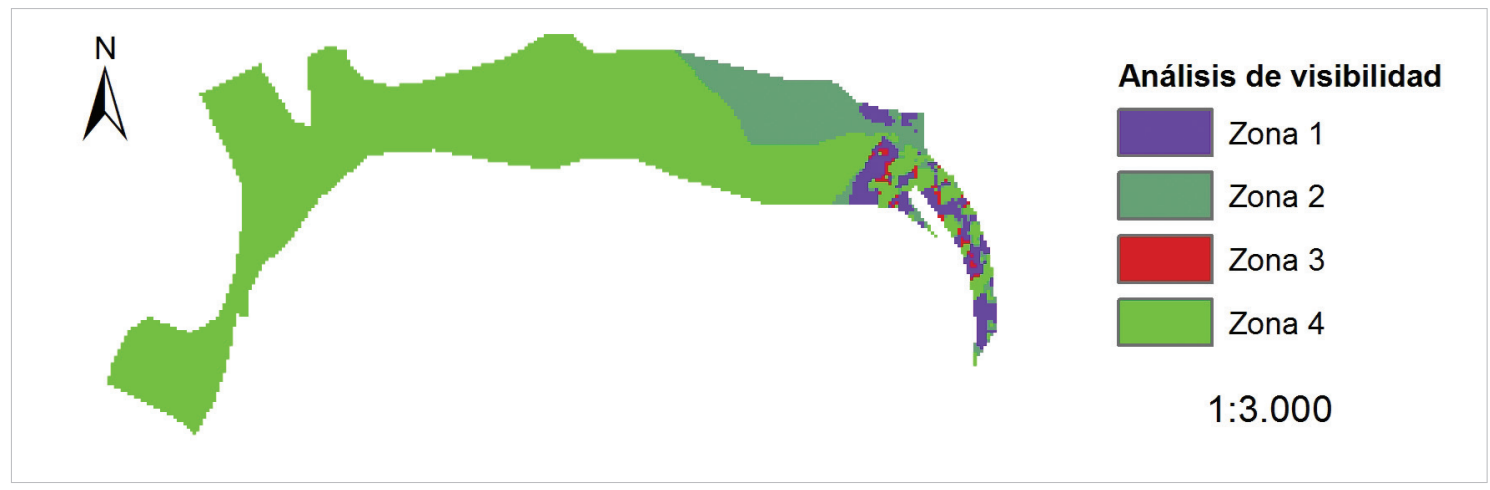

Figura 6. Resultado del análisis de la visibilidad.

Tabla 1. Visibilidad por zonas.

ZONA OBS1 OBS2 OBS3 OBS4 OBS5 OBS6 OBS7 OBS8 OBS9 OBS10 OBS11

\begin{tabular}{llllllllllll}
\hline 1 & 0 & 0 & 0 & 0 & 0 & 0 & 0 & 0 & 0 & 0 & 0 \\
\hline 2 & 1 & 0 & 0 & 0 & 0 & 0 & 0 & 0 & 0 & 0 & 0 \\
\hline 3 & 0 & 1 & 0 & 0 & 0 & 0 & 0 & 0 & 0 & 0 & 0 \\
\hline 4 & 1 & 1 & 0 & 0 & 0 & 0 & 0 & 0 & 0 & 0 & 0 \\
\hline
\end{tabular}

\section{Discusión}

Con el uso de la herramienta "Observer Points" del programa ArcGIS 10.1 podemos realizar las cuencas visuales desde varios puntos de observación en un solo paso y obtener la cuenca visual acumulada sin tener que realizar otros tipos de procesamientos en el sistema de información geográfica. El estado idóneo de los datos permite realizar modelos digitales de superficie más precios a diferencia de si presentan errores.

\section{Conclusiones}

El uso de modelos digitales de superficie aporta al análisis visual de los estudios de integración paisajística mayor precisión debido a que incorporan los elementos presentes en el medio físico (vegetación, construcciones, edificaciones, etc.), ya que estos interceden en la visión del observador. También se obtiene mayor realidad en la representación realística en 3D en comparación con el uso de modelos digitales del terreno.

Los datos LiDAR pueden presentar errores tanto a la falta de datos/tratamiento en los archivos LAZ como errores debido a outlayers, en el primer caso no se puede subsanar el error, mientras que para los outlayers estos pueden ser eliminados de los datos originales para obtener un modelo digital de superficie de mayor calidad. 
Una de las limitaciones que puede producirse en el uso de los datos LiDARse debe a que los árboles a la hora de realizar la cuenca visual aparecen en forma de montículos, no existiendo una permeabilidad visual a través de ellos. En este caso se ha trabajo una masa forestal, por lo que esta limitación afectaría de forma leve ya que los árboles están en pendiente y en espesura, produciendo una barrera en su conjunto. Otra limitación es debida la modificación del paisaje desde la toma de los datos LiDAR hasta la realización del análisis visual, ya que si se produjesen cambios debidos a nuevas construcciones, aprovechamientos forestales, incendios forestales, etc. el resultado de la cuenca visual sería distinto.

\section{Bibliografía}

Hamilton, S. E., \& Morgan, A. (2010). Integrating lidar, GIS and hedonic price modeling to measure amenity values in urban beach residential property markets. Computers, Environment and Urban Systems, 34(2), 133-141. http://doi.org/10.1016/j.compenvurbsys.2009.10.007

Klouček, T., Lagner, O., \& Š́mová, P. (2015). How does data accuracy influence the reliability of digital viewshed models? A case study with wind turbines. Applied Geography, 64, 46-54. http://doi.org/10.1016/j.apgeog.2015.09.005

Lindsey, G., Wilson, J., Yang, J. A., Alexa, C., Anne Yang, J., \& Alexa, C. (2008). Urban greenways, trail characteristics and trail use: Implications for design. Journal of Urban Design, 13(1), 53-79. http://doi.org/10.1080/13574800701804033

Lonergan, C., Hedley, N., \& Clague, J.J. (2015). A visibility-based assessment of tsunami evacuation signs in Seaside, Oregon. Natural Hazards, 78(1), 41-59. http://doi.org/ 10.1007/s11069-015-1696-8

McGaughey, R. J. (2009). FUSION/LDV: Software for LiDAR data analysis and visualization. US Department of Agriculture, Forest Service, Pacific Northwest Research Station: Seattle, WA, USA, 123(2).

Murgoitio, J. J., Shrestha, R., Glenn, N. F., Spaete, L. P., Murgoitio, J. J., Shrestha, R., ... Spaete, L. P. (2013). Improved visibility calculations with tree trunk obstruction modeling from aerial LiDAR. International Journal of Geographical Information Science, 27 (10), 1865-1883. http://doi.org/10.1080/13658816.2013.767460

Wilson, J., Lindsey, G., \& Liu, G. (2008). Viewshed characteristics of urban pedestrian trails, Indianapolis, Indiana, USA. Journal of Maps, 4(1), 108-118. http://doi.org/10.4113/jom. 2008.1014 
